\title{
Kaon decays at NA48: recent results and perspectives
}

\author{
Andrea Bizzeti ${ }^{* \dagger}$ \\ Dipartimento di Scienze Fisiche, Informatiche e Matematiche \\ dell'Università di Modena e Reggio Emilia - via G. Campi 213/A, I-41125 Modena, Italy \\ and I.N.F.N., Sezione di Firenze - via G. Sansone 1, I-50018 Sesto Fiorentino (FI), Italy \\ E-mail: andrea.bizzeti@unimore.it
}

We report recent results on charged kaon rare decays obtained by the NA48/2 collaboration.

The $\mathrm{K}_{\mathrm{e} 4}$ decays $K^{ \pm} \rightarrow \pi^{+} \pi^{-} e^{ \pm} \boldsymbol{v}\left(\mathrm{K}_{\mathrm{e} 4}(+-)\right)$ and $K^{ \pm} \rightarrow \pi^{0} \pi^{0} e^{ \pm} \boldsymbol{v}\left(\mathrm{K}_{\mathrm{e} 4}(00)\right)$ have been studied extensively, providing measurements of the branching ratios, together with the $\mathrm{K}_{\mathrm{e} 4}(+-)$ form factors and a precise determination of $\pi \pi$ scattering lengths. Prospects for a study of $\mathrm{K}_{\mu 4}$ decays are discussed.

A new measurement of the branching ratio and $\gamma \gamma$ invariant mass spectrum of $K^{ \pm} \rightarrow \pi^{ \pm} \gamma \gamma$ decays has been obtained from a combined analysis of NA48/2 and NA62 minimum-bias data.

The analysis of a high statistics and low background sample of $K^{ \pm} \rightarrow \pi^{ \pm} \pi^{0} \gamma$ decays provided the first evidence of a non-zero interference between Inner Bremsstrahlung and Direct Emission amplitudes. The $K^{ \pm} \rightarrow \pi^{ \pm} \pi^{0} e^{+} e^{-}$decay has been observed for the first time, with a statistics of about 4500 events.

The branching ratios of $K^{ \pm} \rightarrow \pi^{ \pm} e^{+} e^{-}$and $K^{ \pm} \rightarrow \pi^{ \pm} \mu^{+} \mu^{-}$decays have been measured with a few percent precision. Improved upper limits are given for CP-violating asymmetries in these decays, and for the branching fraction of the Lepton Number violating decay $K^{ \pm} \rightarrow \pi^{\mp} \mu^{ \pm} \mu^{ \pm}$.

The 7th International Workshop on Chiral Dynamics

August 6 -10, 2012

Jefferson Lab, Newport News, Virginia, USA

\footnotetext{
* Speaker.

†n behalf of the NA48/2 collaboration: Cambridge, CERN, Chicago, Dubna, Edinburgh, Ferrara, Florence, Mainz, Northwestern, Perugia, Pisa, Saclay, Siegen, Turin, Vienna
} 


\section{Introduction}

Kaon decays provide unique opportunities to study low energy strong interaction and test the predictions of the Chiral Perturbation Theory.

In this paper we will present recent results from the NA48/2 collaboration on the study of charged kaon rare decays.

\section{The NA48/2 experiment}

The NA48/2 experiment has been taking data in the years 2003 and 2004, detecting in-flight decays of charged kaons in two simultaneous oppositely charged beams of $(60 \pm 3) \mathrm{GeV} / \mathrm{c}$ momentum produced as secondary beams at CERN SPS. The charged particle reconstruction is provided by a magnetic spectrometer, consisting of a dipole magnet and four drift chamber stations, with a spatial resolution of $100 \mu \mathrm{m}$ and a momentum resolution $\Delta p / p=(1.0 \oplus 0.044 p[\mathrm{GeV} / \mathrm{c}]) \%$.

The spectrometer is followed by a scintillator hodoscope consisting of two planes segmented into horizontal and vertical strips, achieving a very good $\sim 150 \mathrm{ps}$ time resolution.

A Liquid Krypton calorimeter ( $\mathrm{LKr}$ ), consisting of a $27 X_{0}$ almost homogeneous ionization chamber with high-granularity tower read-out, is used to measure electromagnetic deposits and identify electrons through their $E / p$ ratio. Its energy resolutions is $\Delta E / E=3.2 \% / \sqrt{E[\mathrm{GeV}]} \oplus$ $9 \% / E[\mathrm{GeV}] \oplus 0.42 \%$, its position resolution about $1.5 \mathrm{~mm}$.

Muon and photon veto counters and an iron-scintillator hadron calorimeter complete the experimental apparatus, a detailed description of which can be found in Ref. [1].

Large data samples have been recorded, containing about $4 \times 10^{9} \mathrm{~K}^{ \pm} \rightarrow \pi^{ \pm} \pi^{+} \pi^{-}$and $10^{8} \mathrm{~K}^{ \pm} \rightarrow$ $\pi^{ \pm} \pi^{0} \pi^{0}$ well reconstructed decays.

\section{The $K^{ \pm} \rightarrow \pi \pi e^{ \pm} v\left(\mathrm{~K}_{\mathrm{e} 4}\right)$ decay}

The kinematics of $K^{ \pm} \rightarrow \pi^{+} \pi^{-} e^{ \pm} v\left(\mathrm{~K}_{\mathrm{e} 4}(+-)\right)$ and $K^{ \pm} \rightarrow \pi^{0} \pi^{0} e^{ \pm} v\left(\mathrm{~K}_{\mathrm{e} 4}(00)\right)$ decays, sketched in Fig. 1, is fully described by the five independent Cabibbo-Maksymowicz variables[2] the di-pion and di-lepton squared invariant masses $s_{\pi}=m_{\pi \pi}^{2}$ and $s_{e}=m_{e V}^{2}$; the angles $\theta_{\pi}\left(\theta_{e}\right)$ in the di-pion (di-lepton) rest frame of $\pi^{ \pm}\left(e^{ \pm}\right)$with respect to the $\pi \pi(e v)$ flight direction in the $K^{ \pm}$rest frame; and the angle $\varphi$ between the $\pi \pi$ and $e v$ decay planes in the $K^{ \pm}$rest frame.

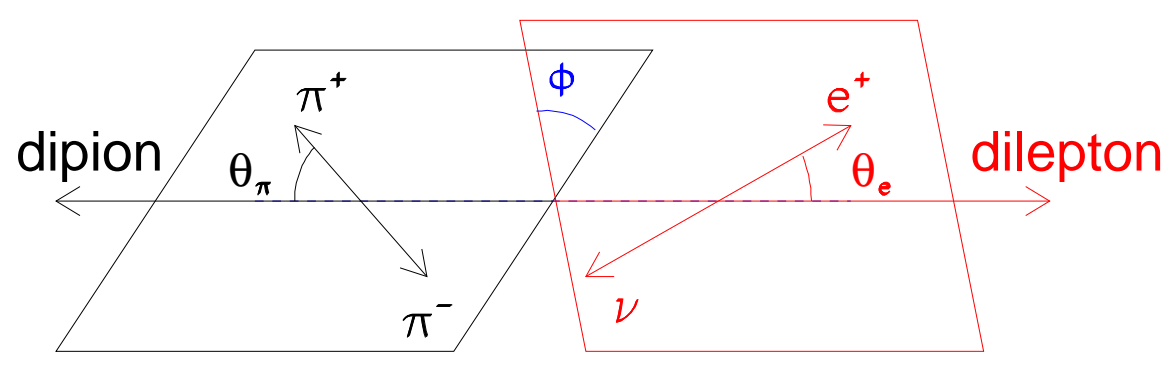

Figure 1: Topology of the $K_{e 4}$ decay showing the angle definitions. 
Form factors can be expanded in partial waves[3]. Limiting the expansion to $\mathrm{S}$ and $\mathrm{P}$ waves and considering a unique phase $\delta_{p}$ for all $\mathrm{P}$-wave form factors, two complex axial $(F, G)$ and one complex vector $(H)$ form factors contribute to the transition amplitude: $F=F_{s} e^{i \delta_{s}}+F_{p} e^{i \delta_{p}} \cos \theta_{\pi}$, $G=G_{p} e^{i \delta_{p}}, H=H_{p} e^{i \delta_{p}}$.

Four real form factors $\left(F_{s}, F_{p}, G_{p}\right.$ and $\left.H_{p}\right)$ and a single phase $\left(\delta=\delta_{s}-\delta_{p}\right)$ have to be measured, including their energy variation. In the $\mathrm{K}_{\mathrm{e} 4}(00)$ mode, the variables $\theta_{\pi}$ and $\phi$ are irrelevant and the form factors reduce to the single $F_{s}$ value due to the Bose statistics. In addition, if $\Delta I=1 / 2$ holds, $F_{S}$ should be equal in both $\mathrm{K}_{\mathrm{e} 4}$ modes.

Each of the $\mathrm{K}_{\mathrm{e} 4}$ branching ratios (BR) is measured relative to a normalization mode $(n)$ as :

$$
\mathrm{BR}\left(\mathrm{K}_{\mathrm{e} 4}\right)=\frac{\left(N_{s}-N_{b}\right)}{N_{n}} \frac{A_{n} \varepsilon_{n}}{A_{s} \varepsilon_{s}} \mathrm{BR}(n)
$$

where $N_{s}, N_{b}, N_{n}$ are the numbers of signal, background and normalization candidates, $A_{s}$ and $\varepsilon_{s}$ are the geometrical acceptance and trigger efficiency for the signal sample, $A_{n}$ and $\varepsilon_{n}$ the geometrical acceptance and trigger efficiency for the normalization sample.

\subsection{Analysis of the $K^{ \pm} \rightarrow \pi^{+} \pi^{-} e^{ \pm} v\left(\mathrm{~K}_{\mathrm{e} 4}(+-)\right)$ decay.}

The $\mathrm{K}_{\mathrm{e} 4}(+-)$ BR is measured relative to the abundant $K^{ \pm} \rightarrow \pi^{+} \pi^{-} \pi^{ \pm}\left(\mathrm{K}_{3 \pi}\right)$ decay mode $\left[\mathrm{BR}\left(\mathrm{K}_{3 \pi}\right)=(5.59 \pm 0.04) \%\right]$ which contains the same number of charged particles in the final state and is recorded concurrently by the same trigger logic. A very large sample of more than one million $\mathrm{K}_{\mathrm{e} 4}(+-)$ decays has been analyzed to measure $\mathrm{S}$-wave $\pi \pi$ scattering lengths with a few percent precision (see section 3.3 below). Form factors values, and their energy dependence, have been obtained[ 4 ] relative to a single overall factor $f_{s}$ which can be determined from the BR value. The energy dependence is described using a series expansion in the dimensionless invariants $q^{2}=\left[\left(S_{\pi} / 4 m_{\pi^{+}}^{2}\right)-1\right]$ and $S_{e} / 4 m_{\pi^{+}}^{2}$ (three terms for $F_{s}$, two terms for $G_{p}$ and one term for $\left.F_{p}, H_{p}\right)$. The coefficients of this series expansion, normalized to $f_{s}$, are reported in table 1 .

\begin{tabular}{|c|rcc||c|ccc|}
\hline & value & stat & syst & & value & stat & syst \\
\hline$f_{s}^{\prime} / f_{s}$ & 0.152 & \pm 0.007 & \pm 0.005 & $g_{p} / f_{s}$ & 0.868 & \pm 0.010 & \pm 0.010 \\
$f_{s}^{\prime \prime} / f_{s}$ & -0.073 & \pm 0.007 & \pm 0.006 & $g_{p}^{\prime} / f_{s}$ & 0.089 & \pm 0.017 & \pm 0.013 \\
$f_{e}^{\prime} / f_{s}$ & 0.068 & \pm 0.006 & \pm 0.007 & & & & \\
\hline$f_{p} / f_{s}$ & -0.048 & \pm 0.003 & \pm 0.004 & $h_{p} / f_{s}$ & -0.398 & \pm 0.015 & \pm 0.008 \\
\hline
\end{tabular}

Table 1: $\mathrm{K}_{\mathrm{e} 4}(+-)$ decay form factors coefficients (see text).

In the BR measurement several requirements were loosened or removed from the event selection of the form factor analysis. Extra accidental track activity and possible accompanying photons were accepted while particle identification requirements were loosened. Many stability checks were performed to ensure the robustness of the procedure and define systematic uncertainties. Out of $\sim 2.5 \times 10^{10}$ total recorded triggers, $1.11 \times 10^{6} \mathrm{~K}_{\mathrm{e} 4}$ candidates were selected, including about 10545 background events, and $1.9 \times 10^{9}$ normalization candidates. The $\mathrm{K}_{\mathrm{e} 4}$ and $\mathrm{K}_{3 \pi}$ geometrical acceptances (resulting from a GEANT3 simulation) have large and similar values of $18.22 \%$ and $24.18 \%$, respectively. Trigger efficiencies are measured using minimum bias control triggers ${ }^{1}$.

\footnotetext{
${ }^{1}$ because of downscaling, control samples have limited statistics
} 
They have high similar values of $98.3 \%\left(\mathrm{~K}_{\mathrm{e} 4}\right)$ and $97.5 \%\left(\mathrm{~K}_{3 \pi}\right)$. The analysis has been performed for each kaon charge ( $\mathrm{K}_{\mathrm{e} 4}^{-}$mode has never been measured before) and the results statistically combined. The details of the common systematic uncertainties are given in the left column of Table 2 . The values of the branching fractions (including radiative $\mathrm{K}_{\mathrm{e} 4}$ decays) are found to be[8]:

$$
\begin{aligned}
& \mathrm{BR}\left(K^{+} \rightarrow \pi^{+} \pi^{-} e^{+} v\right)=\left(4.255 \pm 0.008_{\text {stat }+ \text { time-dependent syst }}\right) \times 10^{-5} \\
& \mathrm{BR}\left(K^{-} \rightarrow \pi^{+} \pi^{-} e^{-} \bar{v}\right)=\left(4.261 \pm 0.011_{\text {stat }+ \text { time-dependent syst }}\right) \times 10^{-5} \\
& \text { combined into } \operatorname{BR}\left(K^{ \pm} \rightarrow \pi^{+} \pi^{-} e^{ \pm} v\right)=\left(4.257 \pm 0.004_{\text {stat }} \pm 0.016_{\text {syst }} \pm 0.031_{\text {ext }}\right) \times 10^{-5}
\end{aligned}
$$

The relative $0.8 \%$ precision of this measurement, dominated by the external uncertainty from the normalization mode, brings a factor $\sim 3$ improvement with respect to the world average value [5], $\mathrm{BR}\left(\mathrm{K}_{\mathrm{e} 4}\right)=(4.09 \pm 0.10) 10^{-5}$, based on two earlier measurements[6, 而. The relative decay rate $\Gamma\left(\mathrm{K}_{\mathrm{e} 4}\right) / \Gamma\left(\mathrm{K}_{3 \pi}\right)=\left(7.615 \pm 0.008_{\text {stat }} \pm 0.028_{\text {syst }}\right) 10^{-4}$ is measured with a $0.4 \%$ relative precision, a factor $\sim 5$ better than the current world average of $(7.31 \pm 0.16) 10^{-4}$.

From the measured $\mathrm{K}_{\mathrm{e} 4}$ branching ratio (3.4), using the values of the average kaon lifetime value $(1.2380 \pm 0.0021) 10^{-8} \mathrm{~s}$ and the CKM matrix element $\left|V_{u s}\right|=0.2252 \pm 0.0009$ from [5], the measurement of the form factors [ $₫$ ] is complemented by the overall normalization factor[8]

$$
f_{s}=F_{s}\left(q^{2}=0, S_{e}=0\right)=5.705 \pm 0.003_{\text {stat }} \pm 0.017_{\text {syst }} \pm 0.031_{\text {ext }}=5.705 \pm 0.035 \quad .
$$

\begin{tabular}{|ll||lc|}
\hline \multicolumn{1}{|c|}{$K^{ \pm} \rightarrow \pi^{+} \pi^{-} e^{ \pm} \nu$} & $(\%)$ & \multicolumn{1}{c|}{$K^{ \pm} \rightarrow \pi^{0} \pi^{0} e^{ \pm} v$} & $(\%)$ \\
\hline Acceptance and beam geometry & 0.18 & Beam geometry & 0.10 \\
Muon vetoing efficiency & 0.16 & Simulation statistics & 0.12 \\
Accidental activity & 0.21 & Form factor dependence & 0.20 \\
Particle identification & 0.09 & Electron identification & 0.10 \\
Background estimate & 0.07 & Background estimate & 0.35 \\
Radiative events modelling & 0.08 & Radiative events modelling & 0.23 \\
Trigger efficiency & 0.11 & Trigger efficiency & 0.80 \\
Simulation statistics & 0.05 & & \\
\hline Total systematics & 0.37 & Total systematics & 0.94 \\
\hline \hline External error $\left[\mathrm{BR}\left(K^{ \pm} \rightarrow \pi^{+} \pi^{-} \pi^{ \pm}\right)\right]$ & 0.72 & External error $\left[\mathrm{BR}\left(K^{ \pm} \rightarrow \pi^{0} \pi^{0} \pi^{ \pm}\right)\right]$ & 1.25 \\
\hline
\end{tabular}

Table 2: Summary of the relative systematic uncertainties on $B R\left(K_{e 4}\right)$ measurements.

\subsection{Analysis of the $K^{ \pm} \rightarrow \pi^{0} \pi^{0} e^{ \pm} v\left(\mathrm{~K}_{\mathrm{e} 4}(00)\right)$ decay.}

The $\mathrm{K}_{\mathrm{e} 4}(00)$ BR is measured relative to the more abundant decay mode $K^{ \pm} \rightarrow \pi^{0} \pi^{0} \pi^{ \pm}$ $\left[\mathrm{BR}\left(\mathrm{K}_{3 \pi}\right)=(1.761 \pm 0.022) \%\right]$, having a very similar final state: one charged particle and two $\pi^{0}$ s detected as four decay photons in the LKr. Both modes are recorded concurrently by the same trigger logic. The event selection and reconstruction follow very closely those developed for the detailed analysis of the normalization mode[ [9]. Normalization events are required to have a low total transverse momentum relative to the beam line $\left(p_{t}\right)$ and reconstruct the $\pi^{0} \pi^{0} \pi^{ \pm}$mass close to the kaon mass when $m_{\pi^{+}}$is assigned to the charged particle, while signal events are required to 
reconstruct the $\pi^{0} \pi^{0} \pi^{ \pm}$mass away from the kaon mass and/or have a sizeable $p_{t}$ with respect to the beam line (Figure 2). Additional requirements on the LKr energy associated to the charged track ( $E / p$ close to 1 and shower properties) ensure electron identification. The dominant background comes from $\mathrm{K}_{3 \pi}$ events with misidentification of the charged pion as an electron. Its contribution can be measured from control regions in the two decay modes. The background from $\mathrm{K}_{3 \pi}$ events with a subsequent $\pi^{ \pm} \rightarrow e^{ \pm} v$ decay has been studied from simulation and contributes one order of magnitude lower.
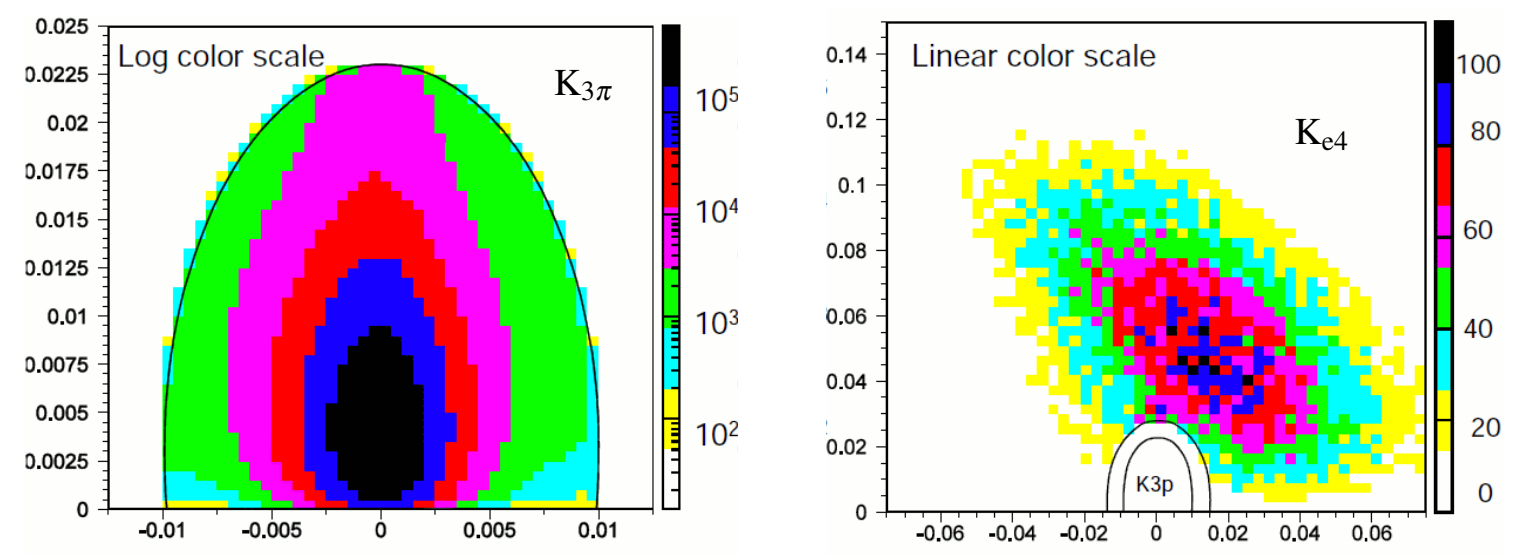

Figure 2: Event distribution in the $\left(\left[M(3 \pi)-M_{K}\right], P_{t}\right)$ plane (units in $\mathrm{GeV} / \mathrm{c}^{2}, \mathrm{GeV} / \mathrm{c}$ ). Left plot: $\mathrm{K}_{3 \pi}$ candidates (normalization). Right plot: $\mathrm{K}_{\mathrm{e} 4}(00)$ candidates (signal); the small ellypse corresponds to the one shown in the left plot; events outside the larger ellypse are selected as signal candidates.

The total background is estimated to be $\sim 1.3 \%$ relative to the signal. Geometrical acceptances have been computed using GEANT3-based simulations, including our best knowledge of the normalization mode[10] which describes accurately the observed cusp effect and using the $\mathrm{K}_{\mathrm{e} 4}(+-)$ measured $F_{S}$ value [8] for the signal simulation. They amount to $4.11 \%$ and $1.77 \%$, respectively. The analysis selected $\sim 71 \times 10^{6}$ normalization events, $44909 \mathrm{~K}_{\mathrm{e} 4}$ candidates and 598 background events. Trigger efficiencies have been measured from minimum bias control triggers. They vary with data taking conditions between 92 and $98 \%$, but the ratio $\varepsilon_{n} / \varepsilon_{s}$ is stable and close to unity. Preliminary systematic uncertainties have been quoted conservatively and are displayed in the right column of Table 2. A preliminary branching ratio value (including radiative $\mathrm{K}_{\mathrm{e} 4}$ decays) for the combined $\mathrm{K}_{\mathrm{e} 4}^{ \pm}(00)$ sample is obtained as:

$$
\mathrm{BR}\left(K^{ \pm} \rightarrow \pi^{0} \pi^{0} e^{ \pm} v\right)=\left(2.595 \pm 0.012_{\text {stat }} \pm 0.024_{\text {syst }} \pm 0.032_{\text {ext }}\right) \times 10^{-5} .
$$

The total error $\pm 0.042 \times 10^{-5}$ (1.6\% relative) is dominated by the external error ( $1.25 \%$ relative). This measurement brings a factor of ten improvement on the total error with respect to the world average $(2.2 \pm 0.4) 10^{-5}$. The final form factor analysis will include a correction for small negative interference of the $\mathrm{K}_{\mathrm{e} 4}(+-)$ mode with final state charge exchange scattering $\left(\pi^{+} \pi^{-} \rightarrow \pi^{0} \pi^{0}\right)$ below $S_{\pi}=\left(2 m_{\pi^{+}}\right)^{2}$ threshold (see Fig. 3).

\subsection{Determination of the $\pi \pi$ scattering lengths}

The value of the $\pi \pi$ scattering lengths $a_{0}$ and $a_{2}$ (corresponding to isospin states $\mathrm{I}=0$ and $\mathrm{I}=2$, 

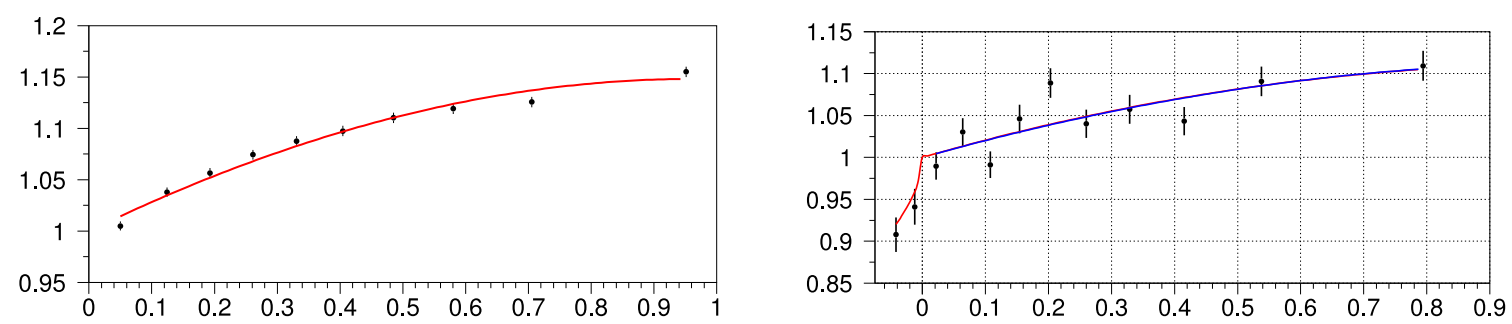

Figure 3: NA48/2 measurement of $F_{s}$ form factor as a function of $q^{2}=S_{\pi} /\left(4 m_{\pi^{+}}^{2}\right)-1$, in: (left) $\mathrm{K}_{\mathrm{e} 4}(+-)$ decays; (right) $\mathrm{K}_{\mathrm{e} 4}(00)$ decays. The slope variation at $q^{2}=0$ in $\mathrm{K}_{\mathrm{e} 4}(00)$ decays is interpreted as an effect of the interference with the $\mathrm{K}_{\mathrm{e} 4}(+-)$ mode with $\pi^{+} \pi^{-} \rightarrow \pi^{0} \pi^{0}$ final state rescattering.

respectively) are accurately predicted by the standard Chiral Perturbation Theory[11] to be:

$$
a_{0} m_{\pi^{+}}=0.220 \pm 0.005 ; \quad a_{2} m_{\pi^{+}}=-0.0444 \pm 0.0010 ; \quad\left(a_{0}-a_{2}\right) m_{\pi^{+}}=0.264 \pm 0.004 .
$$

Two independent measurements [4 9] of these scattering lengths have been performed by the NA48/2 collaboration from the analysis of $\mathrm{K}_{\mathrm{e} 4}$ and $\mathrm{K}_{3 \pi}$ decays.

The $\mathrm{K}_{\mathrm{e} 4}$ decay amplitude depends on the difference $\delta=\delta_{0}-\delta_{1}$ between the S- and P-wave phase shifts in isosinglet ( $\mathrm{I}=0) \pi \pi$ scattering. The very clean environment due to the absence of other hadrons in the final state allows a precise determination of $\pi \pi$ scattering lengths.

Numerical solutions [12, 13] of the Roy equations [14] can be used to predict the value of the phase $\delta$ as a function of $S_{\pi}$ for any value of $a_{0}$ and $a_{2}$. The scattering lengths $a_{0}$ and $a_{2}$ are extracted performing a 2-parameter fit on the measured values of the phase $\delta$ near threshold. The resulting values[ $[\rrbracket$ of the scattering lengths are:

$$
\begin{aligned}
& a_{0} m_{\pi^{+}}=0.2220 \pm 0.0128_{\text {stat }} \pm 0.0050_{\text {syst }} \pm 0.0037_{\text {ext }} \\
& a_{2} m_{\pi^{+}}=-0.0432 \pm 0.0086_{\text {stat }} \pm 0.0034_{\text {syst }} \pm 0.0028_{\text {ext }}
\end{aligned}
$$

with a large correlation coefficient $\rho=0.969$. Effects from isospin symmetry breaking [15] $\left(m_{\pi^{+}} \neq\right.$ $m_{\pi^{0}}, m_{u} \neq m_{d}$ ) on the phase shift difference $\delta$ have been properly taken into account.

The other measurement of the $\pi \pi$ scattering lengths is based on the analysis of the $K^{ \pm} \rightarrow$ $\pi^{0} \pi^{0} \pi^{ \pm}\left(\mathrm{K}_{3 \pi}\right)$ decays. A cusp-like anomaly in the $\pi^{0} \pi^{0}$ invariant mass $\left(M_{00}\right)$ spectrum in the region close to $2 m_{\pi^{+}}$has been observed for the first time[9] in an analysis of a partial sample of $2.4 \cdot 10^{7}$ NA48/2 events. This anomaly is theoretically described[17, 18] as the effect of the interference with the $K^{ \pm} \rightarrow \pi^{+} \pi^{-} \pi^{ \pm}$decay followed by a $\pi^{+} \pi^{-} \rightarrow \pi^{0} \pi^{0}$ final state rescattering.

The scattering lengths $a_{0}$ and $a_{2}$ are determined from the full NA48/2 data sample, fitting the $M_{00}$ spectrum to theoretical predictionsl[19, 20] for different values of $a_{0}$ and $a_{2}$.

The final NA48/2 result[9] on the $\pi \pi$ scattering lengths from $K^{ \pm} \rightarrow \pi^{0} \pi^{0} \pi^{ \pm}$decays is:

$$
\begin{aligned}
\left(a_{0}-a_{2}\right) m_{\pi^{+}} & =0.2571 \pm 0.0048_{\text {stat }} \pm 0.0025_{\text {syst }} \pm 0.0014_{\text {ext }} \\
a_{2} m_{\pi^{+}} & =-0.024 \pm 0.013_{\text {stat }} \pm 0.009_{\text {syst }} \pm 0.002_{\text {ext }}
\end{aligned}
$$

with a correlation coefficient $\rho=-0.774$.

The NA48/2 high-precision analyses of $\mathrm{K}_{\mathrm{e} 4}$ and $\mathrm{K}_{3 \pi}$ data provide complementary information on the scattering lengths, with independent experimental uncertainties and different theoretical 
inputs. They can be combined (see Fig. (1) to get an even more precise result:

$$
\begin{aligned}
a_{0} m_{\pi^{+}} & =0.2210 \pm 0.0047_{\text {stat }} \pm 0.0040_{\text {syst }} \\
a_{2} m_{\pi^{+}} & =-0.0429 \pm 0.0044_{\text {stat }} \pm 0.0028_{\text {syst }} \\
\left(a_{0}-a_{2}\right) m_{\pi^{+}} & =-0.2639 \pm 0.0020_{\text {stat }} \pm 0.0015_{\text {syst }}
\end{aligned}
$$

in excellent agreement with the values predicted by the standard ChPT[11].

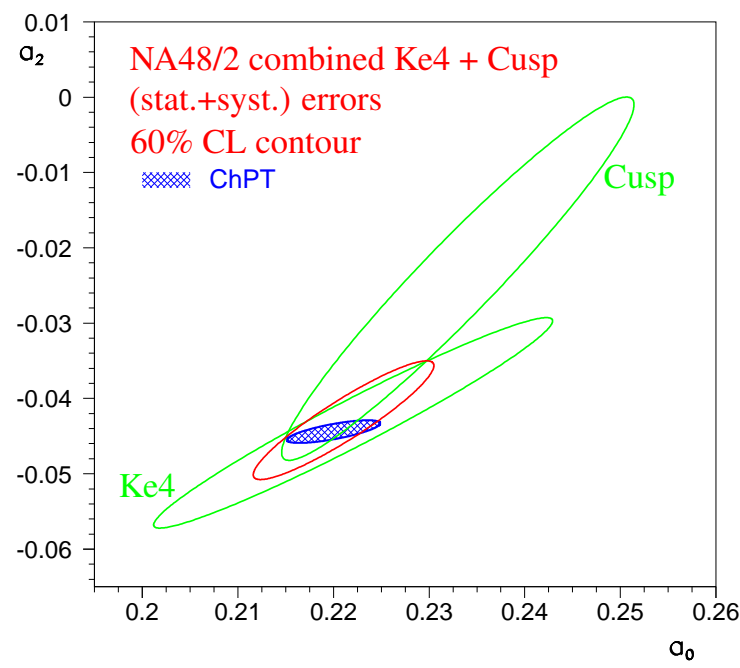

Figure 4: NA48/2 $\mathrm{K}_{\mathrm{e} 4}$ and $\mathrm{K}_{3 \pi}$ (cusp) results as 2-parameter fits in the $\left(a_{0}, a_{2}\right)$ plane (large green ellypses), together with the combined NA48/2 result (smaller red ellypse). The prediction of Chiral Perturbation Theory[1] is shown as a small shadowed blue ellypse. The numbers are in units of $1 / m_{\pi^{+}}$.

\section{The $K^{ \pm} \rightarrow \pi \pi \mu^{ \pm} v\left(\mathrm{~K}_{\mu 4}\right)$ decays}

The $K^{ \pm} \rightarrow \pi^{+} \pi^{-} \mu^{ \pm} v\left(\mathrm{~K}_{\mu 4}(+-)\right)$ and $K^{ \pm} \rightarrow \pi^{0} \pi^{0} \mu^{ \pm} \boldsymbol{v}\left(\mathrm{K}_{\mu 4}(00)\right)$ decays are similar to the the corresponding $\mathrm{K}_{\mathrm{e} 4}$ decays and are described in terms of the same form factors, with an addition of a vector form factor $R$ (vanishing in $\mathrm{K}_{\mathrm{e} 4}$ due to the small electron mass). The Chiral perturbation theory allows to predict [21] the form factors and branching ratio of this decay.

However, the experimental knowledge of the $\mathrm{K}_{\mu 4}$ decay is presently very poor, with only 9 $\mathrm{K}_{\mu 4}(+-)$ events observed until now [22, 23, 24], while the $\mathrm{K}_{\mu 4}(00)$ has not yet been observed. The uncertainty on the measured branching ratio of the $\mathrm{K}_{\mu 4}(+-)$ decay $\mathrm{BR}=(1.4 \pm 0.9) \times 10^{-5}$ is much larger than that on the theoretical prediction $\mathrm{BR}=(0.412 \pm 0.018) \times 10^{-5}$.

From the several thousands of $\mathrm{K}_{\mu 4}(+-)$ events and few thousands of $\mathrm{K}_{\mu 4}(00)$ events expected in the NA48/2 data we plan to measure both their branching ratios and try a first attempt to measure the $R$ form factor.

\section{The $K^{ \pm} \rightarrow \pi^{ \pm} \gamma \gamma$ decay}

Measurements of radiative non-leptonic kaon decays provide crucial tests for the ability of the Chiral Perturbation Theory (ChPT) to explain weak low energy processes. In the ChPT framework, 
the $K^{ \pm} \rightarrow \pi^{ \pm} \gamma \gamma$ decay (denoted $K_{\pi \gamma \gamma}$ below) is precisely predicted as a function of an unknown $O(1)$ constant $\hat{c}$ representing the total contribution of the counterterms in the loop amplitude. The expected di-photon invariant mass $\left(m_{\gamma \gamma}\right)$ spectrum exhibits a characteristic cusp at twice the charged pion mass. Higher order unitarity corrections from $K \rightarrow 3 \pi$ decays $\left(O\left(p^{6}\right)\right.$ and above) modify the lowest order $O\left(p^{4}\right)$ decay spectrum significantly: in particular, they lead to a non-zero differential decay rate at $m_{\gamma \gamma}=0$ [25]. The total decay rate is predicted to be $\operatorname{BR}\left(K_{\pi \gamma \gamma}\right) \sim 10^{-6}$ [26, 27].

The only published experimental $K_{\pi \gamma \gamma}$ observation is that of $31 K^{+}$decay candidates in the kinematic region $100 \mathrm{MeV} / \mathrm{c}<p_{\pi}^{*}<180 \mathrm{MeV} / \mathrm{c}$, where $P_{\pi}^{*}$ is the $\pi^{+}$momentum in the $K^{+}$frame, by the BNL E787 experiment 28].

About $300 K_{\pi \gamma \gamma}$ events have been selected from a minimum bias sample collected by the NA48/2 and NA62 experiments in 2003-2007. A preliminary result on the (model dependent) branching fraction gives $\operatorname{BR}\left(K_{\pi \gamma \gamma}\right)=(1.01 \pm 0.06) 10^{-6}$. The value of the $\hat{c}$ parameter has been derived in both $O\left(p^{4}\right)$ and $O\left(p^{6}\right)$ power expansion of ChPT, obtaining $\hat{c}\left[O\left(p^{4}\right)\right]=1.56 \pm 0.23$ and $\hat{c}\left[O\left(p^{4}\right)\right]=2.00 \pm 0.26$. A detailed description of the analysis is reported in [29].

\section{The $K^{ \pm} \rightarrow \pi^{ \pm} \pi^{0} \gamma^{(*)}$ decays}

The radiative decay $K^{ \pm} \rightarrow \pi^{ \pm} \pi^{0} \gamma^{(*)}$ (where $\gamma^{(*)}$ can be either a real or a virtual photon) is a very interesting channel for studying the low energy structure of QCD. The total amplitude of this decay is the sum of two terms: the dominant one arises from the inner bremsstrahlung (IB) associated to the decay $K^{ \pm} \rightarrow \pi^{ \pm} \pi^{0}$ with the photon emitted from the outgoing charged pion, the other is due to direct emission (DE) in which the photon is radiated in the intermediate states of the decay. Direct photon emission can occur through both electric and magnetic dipole transitions. The electric dipole transition interferes with the IB amplitude giving rise to an interference term (INT), which can have $\mathrm{CP}$ violating contributions. A measurement of both DE and INT terms allows the determination of both the electric and magnetic contributions.

\subsection{Analysis of the $K^{ \pm} \rightarrow \pi^{ \pm} \pi^{0} \gamma$ decay}

The properties of the $K^{ \pm} \rightarrow \pi^{ \pm} \pi^{0} \gamma$ decay can be conveniently described using the $T_{\pi}^{*}$ and $W$ kinematic variables, where $T_{\pi}^{*}$ is the kinetic energy of the charged pion in the kaon rest frame and $W$ is a Lorentz invariant variable given by $W^{2}=\left(P_{K}^{*} \cdot P_{\gamma}^{*}\right)\left(P_{\pi}^{*} \cdot P_{\gamma}^{*}\right) /\left(m_{K}^{2} m_{\pi}^{2}\right)$, where $P_{K}^{*}, P_{\pi}$ and $P_{\gamma}$ are the 4-momenta of the kaon, the charged pion and the photon, respectively. The differential decay width can be written as:

$$
\frac{\partial^{2} \Gamma^{ \pm}}{\partial T_{\pi}^{*} \partial W}=\frac{\partial^{2} \Gamma_{\mathrm{IB}}^{ \pm}}{\partial T_{\pi}^{*} \partial W}\left[1+2 \cos \left( \pm \phi+\delta_{1}^{1}-\delta_{0}^{2}\right) m_{\pi}^{2} m_{K}^{2} X_{E} W^{2}+m_{\pi}^{4} m_{K}^{4}\left(\left|X_{E}\right|^{2}+\left|X_{M}\right|^{2}\right) W^{4}\right]
$$

where $\partial^{2} \Gamma_{\mathrm{IB}}^{ \pm} / \partial T_{\pi}^{*} \partial W$ is the differential rate for the IB component, $\phi$ is the CP violating phase, $\delta_{L}^{I}$ are the strong rescattering phases for a pion-pion state of isospin $I$ and orbital angular momentum $L, X_{E}$ and $X_{M}$ are the normalized electric and magnetic amplitudes of the DE; $\phi, X_{E}$ and $X_{M}$ are constants, while $\delta_{1}^{1}$ and $\delta_{0}^{2}$ are known functions of the $\pi \pi$ invariant mass. The three terms in the sum represent IB, INT and DE contributions, respectively. 
Up to now the DE contribution has only been measured [30, 31, 32, 33] ignoring the INT term, in a limited kinematic range $55 \mathrm{MeV}<T_{\pi}^{*}<90 \mathrm{MeV}$, which is less affected by $K^{ \pm} \rightarrow \pi^{ \pm} \pi^{0}\left(\pi^{0}\right)$ background $^{2}$.

In order to improve the sensitivity to DE and INT components, events have been selected in a wide kinematic region (defined by $T_{\pi}^{*}<80 \mathrm{MeV}$ and $E_{\gamma}>5 \mathrm{GeV}$ ), containing a large fraction of DE and INT spectra. An almost background-free data sample of $6.0 \cdot 10^{5} K^{ \pm} \rightarrow \pi^{ \pm} \pi^{0} \gamma$ candidate events has been selected.

The measured $W$ distribution has been fitted to the sum of MonteCarlo simulated $W$ spectra for IB, DE and INT. By performing a Poissonian Maximum Likelihood fit in the range $0.2<W<0.9$ we obtain[34] the following values for the fractions of DE and INT, normalized to IB:

$$
\begin{aligned}
& \operatorname{Frac}(\mathrm{DE}) \equiv \mathrm{BR}(\mathrm{DE}) / \mathrm{BR}(\mathrm{IB})=\left(3.32 \pm 0.15_{\text {stat }} \pm 0.14_{\text {syst }}\right) \cdot 10^{-2} \\
& \operatorname{Frac}(\mathrm{INT}) \equiv \mathrm{BR}(\mathrm{INT}) / \mathrm{BR}(\mathrm{IB})=\left(-2.35 \pm 0.35_{\text {stat }} \pm 0.39_{\text {syst }}\right) \cdot 10^{-2}
\end{aligned}
$$

with a correlation coefficient $\rho=-0.93$.

The CP-violating asymmetry in the decay rates of $K^{+}$and $K^{-}$is defined by $A_{N} \equiv\left(\Gamma^{+}-\right.$ $\left.\Gamma^{-}\right) /\left(\Gamma^{+}+\Gamma^{-}\right)$, where $\Gamma^{+}$and $\Gamma^{-}$are the $K^{+} \rightarrow \pi^{+} \pi^{0} \gamma$ and $K^{-} \rightarrow \pi^{-} \pi^{0} \gamma$ partial decay widths. In order to measure this asymmetry, the event selection has been modified by lowering the minimum photon energy to $3 \mathrm{GeV}$ and removing the $W>0.2$ cut. We obtain a rate asymmetry $A_{N}=(0.0 \pm$ $\left.1.0_{\text {stat }} \pm 0.6_{\text {syst }}\right) \cdot 10^{-3}$, corresponding to a $90 \%$ CL limit $\left|A_{N}\right|<1.5 \cdot 10^{-3}$.

\subsection{First observation of the $K^{ \pm} \rightarrow \pi^{ \pm} \pi^{0} e^{+} e^{-}$decay}

The main contribution to the $K^{ \pm} \rightarrow \pi^{ \pm} \pi^{0} e^{+} e^{-}$decay amplitude arises from a $\pi^{ \pm} \pi^{0} \gamma^{*}$ intermediate state, with the same three terms (bremsstrahlung, direct emission and its interference) already described in the previous section.

Short-distance effects, sensitive to possible contributions of physics beyond the Standard Model, are expected to produce measurable asymmetries in this decay[35].

The NA48/2 collaboration searched for this decay in the data collected in the years 2003 and 2004. Fig. 5 shows the invariant mass distribution of the $\pi^{ \pm} \pi^{0} e^{+} e^{-}$candidates. The signal peak around the $K^{+}$mass (region between the vertical arrows) contains about 4500 events, and represents the first observation of the $K^{ \pm} \rightarrow \pi^{ \pm} \pi^{0} e^{+} e^{-}$decay.

The main background in the low mass regions is due to $K^{ \pm} \rightarrow \pi^{ \pm} \pi^{0} \pi_{D}^{0}$ decays, where $\pi_{D}^{0} \rightarrow$ $e^{+} e^{-} \gamma$ and the photon is not observed, while the main background in the high mass region comes from $K^{ \pm} \rightarrow \pi^{+} \pi_{D}^{0}$ decays combined with and an accidental photon. The analysis is in progress.

\section{The $K^{ \pm} \rightarrow \pi^{ \pm} e^{+} e^{-}$and $K^{ \pm} \rightarrow \pi^{ \pm} \mu^{+} \mu^{-}$decays}

The large NA48/2 data sample collected in 2003-2004 to measure charge asymmetries in $K^{ \pm} \rightarrow 3 \pi$ decays[36] gives the opportunity to study the $K^{ \pm} \rightarrow \pi^{ \pm} e^{+} e^{-}$and $K^{ \pm} \rightarrow \pi^{ \pm} \mu^{+} \mu^{-}$rare decays and to search for the Lepton Number Violating (LNV) decay $K^{ \pm} \rightarrow \pi^{\mp} \mu^{ \pm} \mu^{ \pm}$.

Three-track vertices are reconstructed extrapolating the segments from the spectrometer, taking into account the measured residual magnetic fields and multiple scattering. For each track, the

\footnotetext{
${ }^{2}$ This cut is effective because $T_{\pi}^{*}\left(K^{ \pm} \rightarrow \pi^{ \pm} \pi^{0}\right) \sim 110 \mathrm{MeV}$ and $T_{\pi}^{*}\left(K^{ \pm} \rightarrow \pi^{ \pm} \pi^{0}\right)<53 \mathrm{MeV}$.
} 


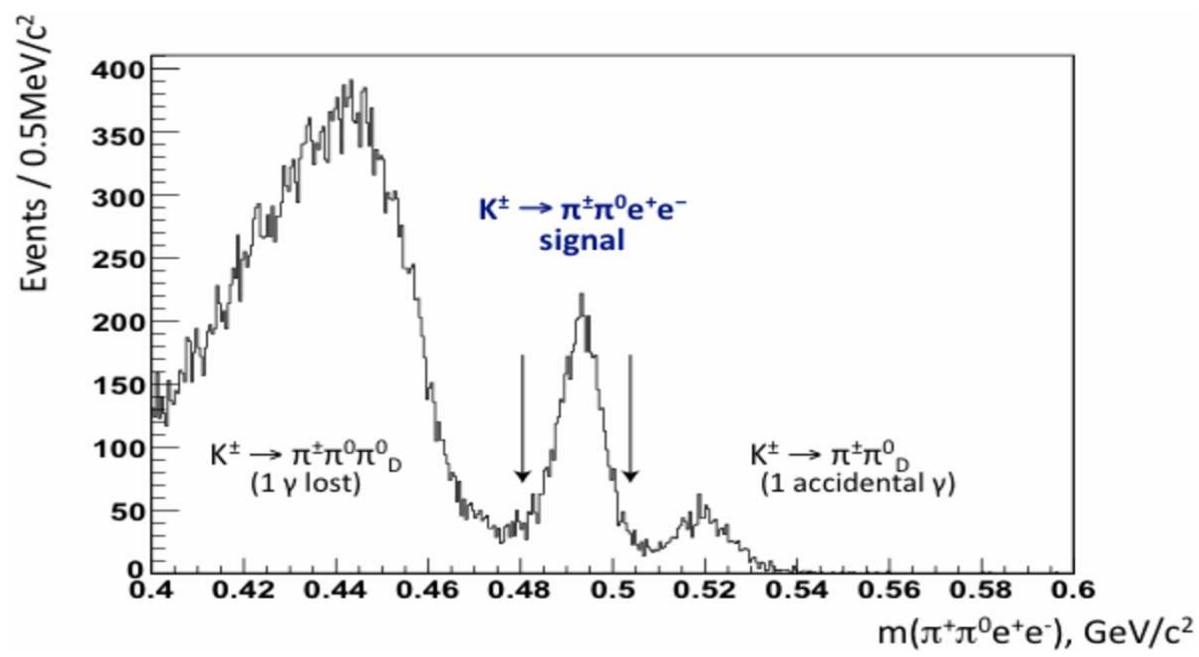

Figure 5: Invariant mass distribution of $K^{ \pm} \rightarrow \pi^{ \pm} \pi^{0} e^{+} e^{-}$candidate events (preliminary). The arrows indicate the boundaries of the signal region around the $K^{+}$mass.

ratio $E / p$ of the associated energy deposit in the $\mathrm{LKr}$ calorimeter to its momentum measured by the magnetic spectrometer is used for particle identification, as described below.

\subsection{The $K^{ \pm} \rightarrow \pi^{ \pm} e^{+} e^{-}$decay}

The $K^{ \pm} \rightarrow \pi^{ \pm} e^{+} e^{-}$rate is measured relative to the more abundant $K^{ \pm} \rightarrow \pi^{ \pm} \pi_{D}^{0}$ normalization channel (where $\pi_{D}^{0} \rightarrow e^{+} e^{-} \gamma$ is the so-called Dalitz decay), whose final state contains the same charged particles as the signal events.

Candidate events are required to have one $\pi^{ \pm} \operatorname{track}(E / p<0.85)$ and a pair of oppositely charged electrons $(E / p>0.95)$; their total momentum is required to be consistent with that of beam particles. Kinematic suppression of the main background channel $\left(K^{ \pm} \rightarrow \pi^{ \pm} \pi_{D}^{0}\right)$ is achieved by requiring the $e^{+} e^{-}$invariant mass to be above the $\pi^{0}$ mass: $z \equiv\left(M_{e e} / M_{K}\right)^{2}>0.08$. Finally, the $\pi^{ \pm} e^{+} e^{-}$invariant mass is required to be consistent with the $K^{ \pm}$mass.

A total of 7253 events has been obtained, with a $1.0 \%$ background.

The branching fraction has been measured to be $\mathrm{BR}\left(K^{ \pm} \rightarrow \pi^{ \pm} e^{+} e^{-}\right)=(3.11 \pm 0.12) \times 10^{-7}$. The first simultaneous observation of both charged kaon decays into $\pi^{ \pm} e^{+} e^{-}$allows to establish an upper limit for the $\mathrm{CP}$ violating charge asymmetry $A_{\mathrm{CP}}=\left(\mathrm{BR}^{+}-\mathrm{BR}^{-}\right) /\left(\mathrm{BR}^{+}+\mathrm{BR}^{-}\right):\left|A_{\mathrm{CP}}\right|<$ $2.1 \times 10^{-2}$ at $90 \% \mathrm{CL}$. More details on this analysis can be found in[37].

\subsection{The $K^{ \pm} \rightarrow \pi^{ \pm} \mu^{+} \mu^{-}$decay}

The $K^{ \pm} \rightarrow \pi^{ \pm} \mu^{+} \mu^{-}$rate is measured relative to the abundant $K^{ \pm} \rightarrow \pi^{ \pm} \pi^{+} \pi^{-}$normalization channel (denoted $K_{3 \pi}$ below). The $K^{ \pm} \rightarrow \pi^{ \pm} \mu^{+} \mu^{-}$and $K_{3 \pi}$ samples are collected concurrently using the same trigger logic.

Candidate events are required to have one $\pi^{ \pm}$track (with $E / p<0.95$ and no correlated hits in the MUV) and a pair of oppositely charged muons $(E / p<0.2$ and associated hit(s) in the MUV); their total momentum is required to be consistent with that of beam particles. The $\pi^{ \pm} \mu^{+} \mu^{-}$ invariant mass is required to be consistent with the $K^{ \pm}$mass (see Fig. 6(left)). 

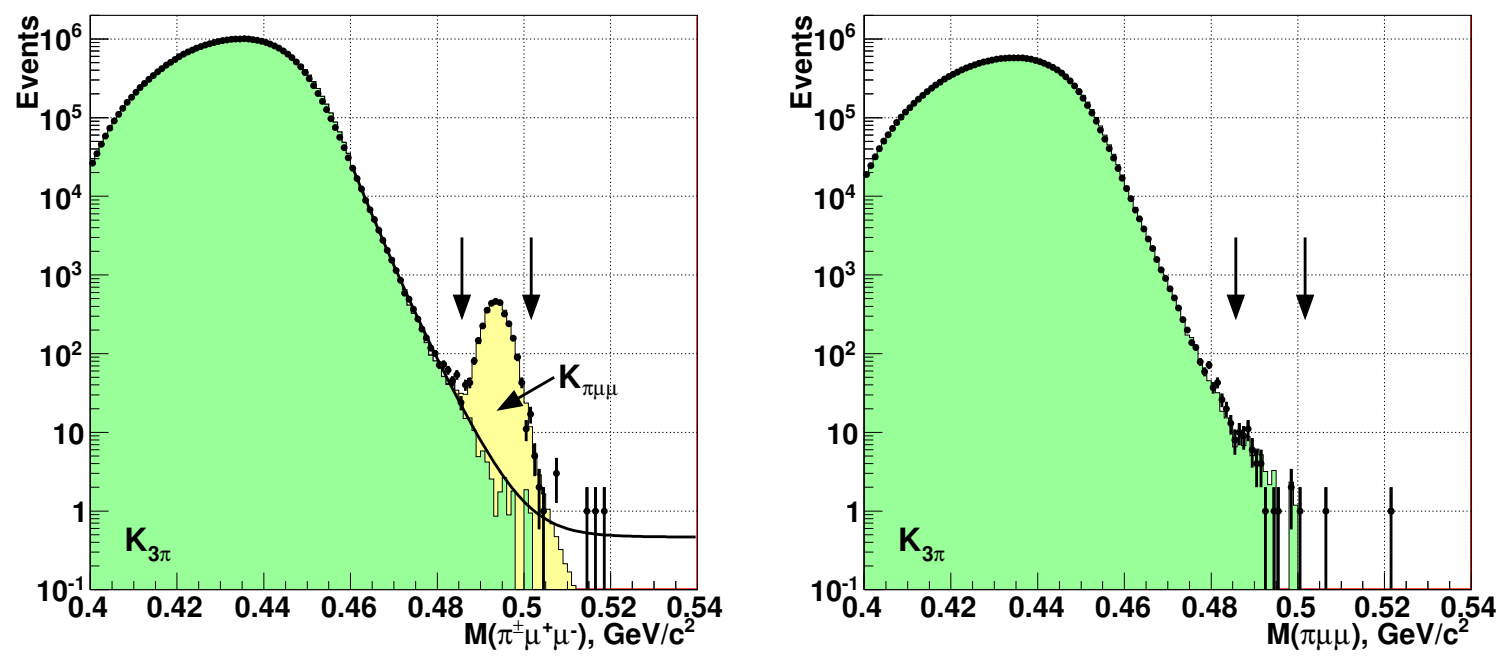

Figure 6: Invariant mass distribution of (left) $\pi^{ \pm} \mu^{+} \mu^{-}$and (right) $\pi^{\mp} \mu^{ \pm} \mu^{ \pm}$candidates: data (dots), $K_{3 \pi}$ and $K_{\pi \mu \mu}$ simulations (filled areas). Arrows indicate the signal region.

A total of 3120 events has been obtained, with a background of $(3.3 \pm 0.7) \%$. The branching fraction has been measured to be $\operatorname{BR}\left(K^{ \pm} \rightarrow \pi^{ \pm} \mu^{+} \mu^{-}\right)=(9.62 \pm 0.25) \times 10^{-8}$, improving the precision by a factor $\sim 3$ with respect to the most precise earlier measurement. The branching ratios have been measured separately for $K^{+}$and $K^{-}$, allowing to establish an upper limit on the $\mathrm{CP}$ violating charge asymmetry $A_{\mathrm{CP}}=\left(\mathrm{BR}^{+}-\mathrm{BR}^{-}\right) /\left(\mathrm{BR}^{+}+\mathrm{BR}^{-}\right):\left|A_{\mathrm{CP}}\right|<2.9 \times 10^{-2}$ at $90 \%$ CL.

The forward-backward asymmetry $A_{\mathrm{FB}}=\frac{\Gamma\left(\cos \theta_{\mathrm{K} \mu}>0\right)-\Gamma\left(\cos \theta_{\mathrm{K} \mu}<0\right)}{\Gamma\left(\cos \theta_{\mathrm{K} \mu}>0\right)+\Gamma\left(\cos \theta_{\mathrm{K} \mu}<0\right)}$ in the angle $\theta_{K \mu}$ between the kaon and the opposite-sign lepton in the di-lepton rest frame has also been measured, resulting in an upper limit $\left|A_{\mathrm{FB}}\right|<2.3 \times 10^{-2}$ at $90 \% \mathrm{CL}$. More details on this analysis can be found in[38].

\subsection{Search for lepton number violation}

The same selection criteria described above for $K^{ \pm} \rightarrow \pi^{ \pm} \mu^{+} \mu^{-}$decays have been used to select events with same-sign muons.

The $\pi \mu \mu$ invariant mass distribution of the selected $K^{ \pm} \rightarrow \pi^{\mp} \mu^{ \pm} \mu^{ \pm}$candidates is shown in Fig. 6 (right). As in the $K^{ \pm} \rightarrow \pi^{ \pm} \mu^{+} \mu^{-}$sample, the main background comes from $K_{3 \pi}$ decays: a Monte Carlo simulation has given $52.6 \pm 19.8$ background events in the signal region (Kaon mass window) where 52 events are found in the data sample.

Conservatively assuming the expected background to be $(52.6-19.8)=32.8$ events we obtain an upper limit of 32.2 signal events at $90 \% \mathrm{CL}$. This corresponds to an upper limit[38] on the branching fraction $\operatorname{BR}\left(K^{ \pm} \rightarrow \pi^{\mp} \mu^{ \pm} \mu^{ \pm}\right)<1.1 \times 10^{-9}$ at $90 \% \mathrm{CL}$, with a factor 3 improvement over the best previous limit.

\section{References}

[1] V. Fanti et al., Nucl. Instr. Methods A 574, 433 (2007).

[2] N. Cabibbo and A. Maksymowicz, Phys. Rev. 137 (1965) B438; ibidem 168 (1968) 1926. 
[3] A. Pais and S. Treiman, Phys. Rev. 168 (1968) 1858.

[4] J.R. Batley et al., Eur. Phys. J. C 70 (2010) 635.

[5] J. Beringer et al. (Particle Data Group), Phys. Rev. D 86 (2012) 010001.

[6] L. Rosselet et al., Phys. Rev. D 15 (1977) 574.

[7] S. Pislak et al., Phys. Rev. D 67 (2003) 072004.

[8] J.R. Batley et al., Phys. Lett. B 715 (2012) 105.

[9] J.R. Batley et al., Eur. Phys. C 64 (2009) 589.

[10] J.R. Batley et al., Phys. Lett. B 6866 (2010) 101.

[11] G. Colangelo, J. Gasser and H. Leutwyler, Nucl. Phys. B 603 (2001) 125.

[12] B. Ananthanarayan, G. Colangelo, J. Gasser and H. Leutwyler, Phys. Rep. 353 (2001) 207.

[13] S. Descotes, N. Fuchs, L. Girlanda and J. Stern, Eur. Phys. J. C 24 (2002) 469.

[14] S. Roy, Phys. Lett. B 36 (1971) 353.

[15] G. Colangelo, J. Gasser and A. Rusetsky, Eur. Phys. J. C 59 (2009) 777.

[16] J.R. Batley et al., Phys. Lett. B 633 (2006) 173 [hep-ex/ 0511056$].$

[17] P. Budini and L. Fonda, Phys. Rev. Lett. 6 (1961) 419.

[18] N. Cabibbo, Phys. Rev. Lett. 93 (2004) 121801 [hep-ph/ 0405001$].$

[19] N. Cabibbo and G. Isidori, J. High Energy Phys. 0503 (2005) 21 [hep-ph/ 0502130 ].

[20] G. Colangelo, J. Gasser, B. Kubis and A. Rusetsky, Phys. Lett. B 638 (2006) 187 [hep-ph/0604084].

[21] J. Bijnens, G. Colangelo and J. Gasser, Nucl. Phys. B 427 (1994) 427.

[22] D.E. Greiner, W.Z. Osborne and W.H. Barkas, Phys. Rev. Lett. 13 (1964) 294.

[23] D. Cline and W.F. Fry, Phys. Lett. 15 (1965) 293.

[24] V. Bisi et al., Phys. Lett. B 25 (1967) 572.

[25] G. Ecker, A. Pich and E. de Rafael, Nucl. Phys B303 (1988) 665.

[26] G. D’Ambrosio and J. Portolés, Phys. Lett. B386 (1996) 403.

[27] J.-M. Gérard, C. Smith and S. Trine, Nucl. Phys B730 (2005) 1.

[28] P. Kitching et al., Phys. Rev. Lett. 79 (1997) 4079.

[29] T. Spadaro, these proceedings, $\operatorname{BoS}(\mathrm{CD} 12) 057$.

[30] S. C. Adler et al., Phys. Rev. Lett. 85 (2000) 4856.

[31] M. A. Aliev et al., Phys. Lett. B 554 (2003) 7.

[32] M. A. Aliev et al., Eur. Phys. J. C 46 (2006) 61.

[33] V. A. Uvarov et al., Phys. Atom. Nucl. 69, 26 (2006).

[34] J. Batley et al., Eur. Phys. J. 68 (2010) 75.

[35] L. Cappiello, O. Catà, G. D’Ambrosio and Dao-Neng Gao, Eur. Phys. J. C 72 (2012) 1872.

[36] J.R. Batley et al., Eur. Phys. J. C 52 (2007) 875.

[37] J.R. Batley et al., Phys. Lett. B 677 (2009) 246.

[38] J.R. Batley et al., Phys. Lett. B 697 (2011) 107. 